\title{
A 6-month Analysis of Factors Impacting Web Browsing Quality for QoE Prediction
}

\author{
Antoine Saverimoutou ${ }^{a}$, Bertrand Mathieu ${ }^{a}$ and Sandrine Vaton ${ }^{b}$ \\ ${ }^{a}$ Orange Labs - Lannion - France ; \{antoine.saverimoutou; bertrand2.mathieu\}@orange.com \\ ${ }^{b}$ IMT Atlantique - Brest - France : sandrine.vaton@imt-atlantique.fr
}

\section{ARTICLE INFO}

\section{Keywords:}

Web browsing

Big Data Analytics

QoE

Prediction

Machine Learning

\begin{abstract}
A B S T R ACT
Web browsing is one of the main Internet services, which has largely evolved over the last years. It now includes many different components (images in various formats, css, Javascript, Progressive Web Apps, etc.) and a web page is composed of many different resources provided by many servers located in various places. Knowing how web browsing content is delivered is crucial for better understanding the Quality of Experience (QoE) offered to end-users. Detecting the factors impacting this QoE is important to improve them but also to be able to predict the quality in order to anticipate degradations to it and propose remediations, which is the main goal of our study. For this, we present an analysis of a measurement campaign we have performed during 6 months (July 2018 - January 2019) on the Top 10,000 Alexa websites, which helps to identify the main factors which can impact the quality perceived by end-users. This set of factors has been confirmed by a machine learning process (decision tree), which gives as outputs the set of rules to predict the QoE. The evaluation of our decision tree-based model, on the Top 10,000-15,000 Alexa websites, never accessed before, shows that we can correctly predict the web browsing quality an end-user can get.
\end{abstract}

\section{Introduction}

Web browsing is a fast-paced and changing domain and needs to deliver a good experience to end-users since a plethora of applications and services are nowadays accessed through web browsers. The Web was originally designed to deliver static contents but has evolved dramatically towards dynamic web pages offering to end-users a built-in environment for education, gaming, video streaming or social networking [1, 2, 3]. Large service companies (e.g Google, Mozilla, Apple, etc.) are following the trend of new technologies by enabling Virtual Reality, Virtual Machines or IoT (Internet of Things) applications embedded in the web browsers. They are delivered over HTTP/1.1 but also over new transport protocols such as HTTP/2 (Version 2 of the HyperText Transfer Protocol) [4], standardized in 2015, or QUIC (Quick UDP Internet Connection) [5, 6] which is paving its way to standardization, as HTTP/3. Network operators or service providers need to offer the best possible QoS (Quality of Service) to their customers and for this, they deploy Content Delivery Networks (CDN) and proxy-based networking architectures, aiming to improve the web browsing delivery.

The question of measuring the quality when performing web browsing and the need to identify the main factors decreasing or enhancing it is then of prime importance. Measuring web browsing quality needs to be performed in a meticulous manner by taking into account the latest richexposed browsers' functionalities. Based on a large measurement campaign we performed over 6 months (July 2018 - January 2019), our first contribution provides an overview of today's web browsing delivery, including the number of resources, their location, the protocol used, etc. Secondly, we perform a detailed study of these parameters' impact on end-users' perceived quality, in order to highlight the factors which are the most critical for Web browsing. Thirdly, we use Machine Learning techniques to validate the identified factors and through a decision tree model, we identify the thresholds of these factors in order to automatically predict the end-users web browsing quality. Then, the identified set of rules of our model are applied on the Alexa top 10K-15K websites and our model proves to be efficient, predicting correctly more than $90 \%$ of measurements.

The paper is structured as follows: We first remind in section 2 the existing web metrics and related work meant to quantify and qualify web browsing. Through section 3 we introduce how our measurement campaign is performed, section 4 depicts how web browsing content is delivered to endusers and section 5 analyses the impacts of the parameters on web browsing quality. Section 6 presents our rules-based model to predict web browsing quality. We finally conclude in section 7 .

\section{Background and Related Work}

Policies and processing algorithms used by web browsers to render web pages are all different. In order to bring uniform benchmarking indicators, standardization bodies such as the $\mathrm{W} 3 \mathrm{C}$, in collaboration with large service companies, have defined a set of web metrics to measure web page loading better. The Page Load Time ${ }^{1}$ (PLT) is the time between the start of the request and when the entire web page has been loaded. The Resource Timing ${ }^{2}$ provides information upon the downloaded resources unit-wise, such as the transport protocol used, size and type of object or some low level networking information. The Paint Timing ${ }^{3}$ exposes the First Paint (FP) which is the time for a first pixel to appear on the end-user's web browser screen. The Above-The-Fold (ATF)

\footnotetext{
${ }^{1}$ https://www.w3.org/TR/navigation-timing/

${ }^{2}$ https://www.w3.org/TR/resource-timing-2/

${ }^{3}$ https://www.w3.org/TR/paint-timing/
} 
[7] exposes the time needed to fully load the visible surface area of a web page at first glance (making use of W3C information). The TFVR (Time for Full Visual Rendering) [8] exposes the loading time of the visible portion of a website at first glance (without scrolling) by making use of networking information through the web browser's exposed HAR (HTTP Archive). The Speed Index and RUM (Real User Monitoring) expose a score representing the visible surface area occupancy of a web page. The Latency Amplification Factor (LAF) [9] characterizes the content of a web page in terms of how it affects the page load time.

When end-users go to a website, third parties such as advertisement networks can impact web browsing quality $[10,11,12]$. To measure QoE during web browsing sessions, several tools have been developed by the research community. FPDetective [13] uses a PhantomJS ${ }^{4}$ and Chromium based automation insfrastructure, OpenWPM [14] performs automated web browsing driven by Selenium ${ }^{5}$ while supporting stateful and stateless measurements and the Chameleon Crawler $^{6}$ is a Chromium based crawler used for detecting browser fingerprinting. Fourth Party [15] instruments the Mozilla-Firefox browser and Web Xray [16] is a PhantomJS based tool for measuring HTTP traffic. XRay [17] and AdFisher [18] run automated personalization detection experiments and Common Crawl ${ }^{7}$ uses an Apache Nutch based crawler. Kaleidoscope [19] is an automated tool to evaluate web features on a large scale focused on the web page style and page loading times. All these tools (among others) have largely contributed to the research field but when wanting to objectively quantify and qualify web browsing, there is a clear need to finely reproduce an end-user environment and usual interaction with the latest web browser functionalities, which they do not offer. We have thus developed our own tool highlighted in section 3 which offers fine-grained information through the latest implemented web metrics and data obtained from network capture and browser's offered HTTP Archive (HAR). We also collect information regarding the different remote web servers located all over the world, the downloaded resources' type, size and Internet protocol through which they are delivered at different stages of the web page loading process.

Web browsing includes 4 key actors, namely the enduser, the web browser, the network and the remote web server. While some research work $[20,21]$ describes how to qualify user-experience better, other studies investigate the impact of different Internet protocols on web browsing quality $[22,23,24,25,26,27]$. Regarding the network, particular interest is given to the upload or download link when investigating bottlenecks [28, 29, 30] and Naseer and al. [31] propose a tool in order to analyze and better understand the actual configuration parameters employed by today's web servers. The PLT is the de facto used web metric but with the evolution of websites' characteristics, researchers ques-

\footnotetext{
${ }^{4}$ http://phantomjs.org/

${ }^{5}$ https://www.seleniumhq.org/

${ }^{6}$ https://github.com/ghostwords/chameleon

${ }^{7}$ https://commoncrawl.org/
}

tion the versatility and objectiveness of this metric to measure end-user's QoE [32, 33, 34, 35, 36] emphasizing that what the user really sees on the screen should be primarily measured. Other research work proposes ATF browserbased measuring techniques $[37,8]$ in order to measure the websites' visible portion loading time. When studying web browsing performance as a whole, different on-market web browsers, Internet protocols, network bandwidths and types of websites need to be taken into account. We have thus focused our research work by taking into account the mostly used on-market web browsers, studied the impact of different Internet protocols (HTTP/1.1, HTTP/2 or QUIC), used different types of residential network access (ADSL, Wi-Fi, Fiber) and assessed the Top 10,000 Alexa websites. Our measurements include all the web metrics defined by the W3C and the TFVR (Time for Full Visible Rendering) [8].

\section{Measurement Campaign Setup}

Our measurement campaign has been performed with the tool Web View[38]. Web View is a measurement platform whose infrastructure includes probes and a public visualization website ${ }^{8}$ illustrating the obtained measurement results for a set of websites. The Web View probe is a userorientated measurement tool whose main objective is to perform automated web browsing sessions and measure representative information of web pages in order to better qualify and understand web browsing, both in terms of quality and delivery. Each test can be configured with several parameters, such as the web browser, the access network type on which the probe is connected, the list of websites to measure, the preferred transport protocol to get the contents (HTTP/1.1, HTTP/2, QUIC), etc.

For this study, we deployed probes in France, three different desktop machines (CPU Intel Core i5 and 8 GB RAM) connected to three different user-representative residential network accesses, i.e ADSL (10Mbps down, 1Mbps up), Wi-Fi (200Mbps down, 160Mbps up) and FTTH Fiber (800Mbps down, 300Mbps up). The Wi-Fi network access (802.11 $\mathrm{b} / \mathrm{g} / \mathrm{n}, 2.4 \mathrm{Ghz}$ and 450Mbit/s max) is provided by a homebox, connected to the Fiber operational network. Our probes are located in the same room as the Wi-Fi homebox. Two popular web browsers, with different window sizes, were used, namely Google-Chrome (version 63 and 68) and MozillaFirefox (version 56 and 62). The Google-Chrome browser is driven by Chromedriver v.2.36 and v.2.40 and MozillaFirefox by Geckodriver v.0.19 where the main automater is Selenium v.3.14. Both browsers support HTTP/1.1 and HTTP/2, but only Google-Chrome implements QUIC. The automated web browsing sessions were conducted at different times of the day by requesting different Internet protocols. Some Internet protocols might be disabled or blocked by service providers or corporate companies and the need to study what the obtained quality is when an Internet protocol is not available is important. When requesting HTTP/1.1, we deactivate the HTTP/2 and QUIC protocols in the browser; when request-

\footnotetext{
${ }^{8}$ https://webview.orange.com
} 


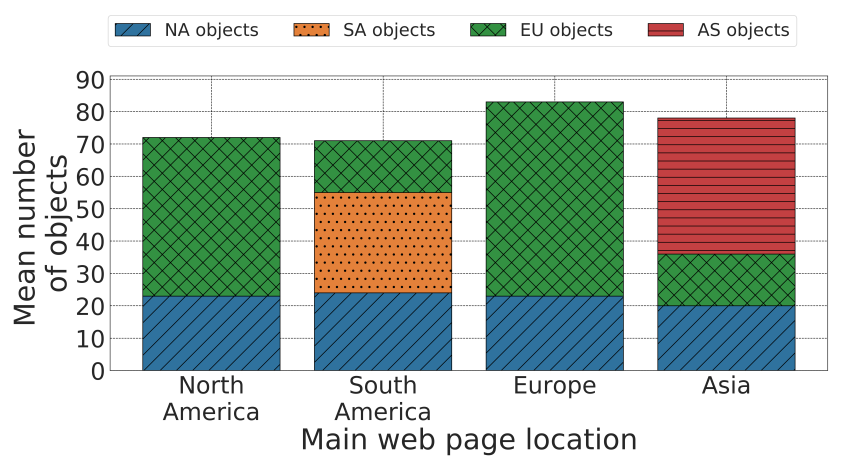

(a) Resources' location

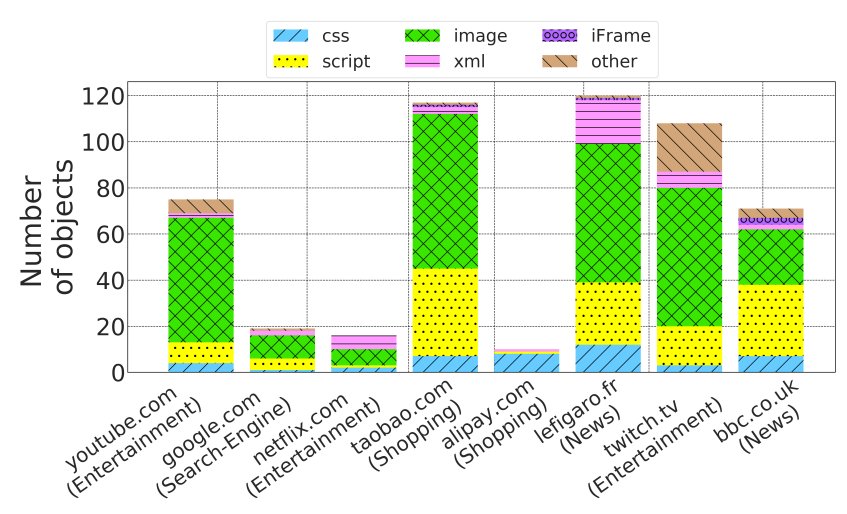

(b) MIME type of resources

Figure 1: Downloaded resources

ing HTTP/2 we deactivate QUIC but allow fallback to HTTP/1. (not all content-servers are HTTP/2-enabled); when requesting QUIC, we allow fallback to HTTP/1.1 and HTTP/2 for non-UDP web servers. We also define another mode, the QUIC Repeat. This mode favors 0-RTT UDP and 1-RTT TCP by firstly performing a navigation to the website, closing the browser, clearing the resources' cache but keeping the DNS (Domain Name System) cache and going once more to the website when we collect measurements. For all our measurements, the resources' cache is always emptied at the start and end of web browsing session, and we have defined a timeout of 18 seconds (this value has been set since it is a very long time to wait for a web page and after more then 18 sec, all end-users will have given up the browsing).

For every visited website, Web View probes measure and compute 84 parameters ${ }^{9}$. Amongst them, we have 4 different loading times, namely the First Paint (FP), Time for Full Visual Rendering (TFVR), the processing time (DOM time) and the Page Load Time (PLT). We also collect network traffic from which we investigate the corresponding DNS time for the main web page domain and through the collected HAR $^{10}$ we assess the overall number of objects downloaded and their MIME (Multipurpose Internet Main Extensions) type. From the exposed remote servers' IP address correlated to MaxMind GeoIP2 database ${ }^{11}$ and RIPE NCC database ${ }^{12}$, we estimate the continents from which these resources are downloaded. Web View also identifies if the resources are delivered with secure connections or not, the number of domains serving the contents and the category of the website as referenced by Alexa. As per the different collected timings, which indicate a web page page loading progression through time, we also collect the number of resources downloaded in these periods of time as well as the Internet protocol through which they are delivered.

After our 6-months campaign on the top 10,000 Alexa

\footnotetext{
${ }^{9}$ https://webview.orange.com/monitoringParameters

${ }^{10}$ HTTP Archive

${ }^{11} \mathrm{http}: / /$ www.maxmind.com

${ }^{12} \mathrm{https}: / /$ stat.ripe.net/
}

websites, we got 244 Million different measurements, representing the measurement of 9597 distinct websites. 403 distinct websites were discarded due to the small amount of web browsing results (these websites were either unavailable, blocked as per our geographic location or not responding in less than 18 seconds). Our dataset then includes measurements for websites having their main web page estimated to be in North America (NA) for $52.23 \%$, in Europe (EU) for $28.44 \%$, in Asia (AS) for $16.22 \%$, and in South America (SA) for $1.10 \%$.

\section{Web Browsing Delivery Analysis}

We present in this section an analysis of our collected measurements and highlight the main evolutions of web browsing delivery with regards to previous studies $[39,9]$ which have identified that the category of a website (as per Alexa ranking), number of objects embedded in the web page and amount of web servers delivering content has an impact on web browsing quality. A part of our dataset is made public at https://webview. orange.com/public/dataset.zip.

\subsection{What are these objects ?}

The web pages are more complex nowadays and we wanted to evaluate their evolution, compared to what it was few years ago $[39,40,41]$. When a main web page is located in Europe, on average between 2 and 47 resources need to be downloaded for less than $25 \%$ of the websites and between 125 and 230 resources need to be downloaded for more than $75 \%$ of these websites. Since 2014, the amount of downloaded resources composing a web page has increased by $17 \%$ on average for the Alexa websites ranked 1-2000 and by $31 \%$ on average for websites ranked 5000-10000.

Going deeper into the question from where the resources are downloaded, (Fig.1a) presents the location of the servers. On average, when a main web page is located in North America, a mean number of 72 resources is downloaded, mainly from EU and from NA. When a main web page is in Asia, an average number of 78 resources is downloaded, about half 


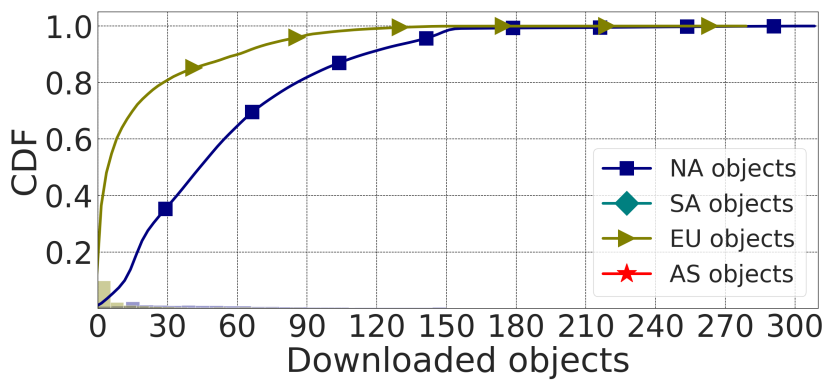

(a) Main web page in NA

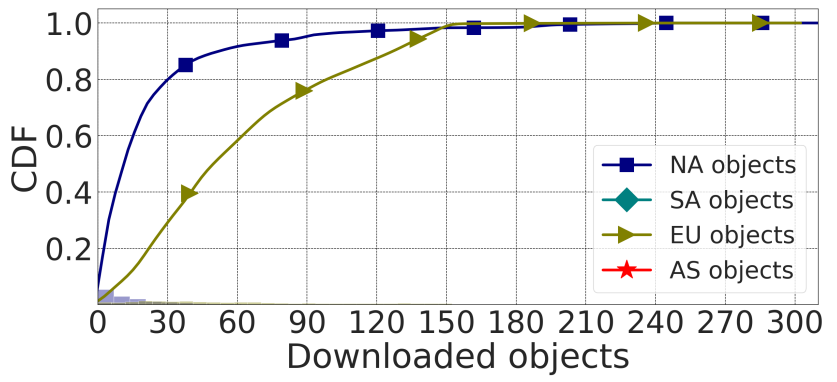

(c) Main web page in EU

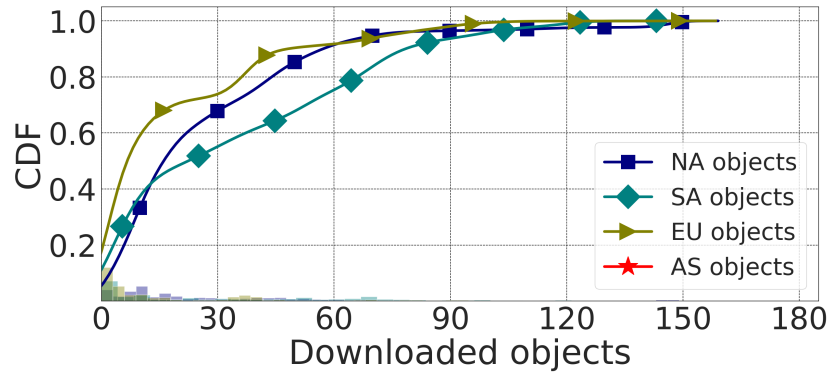

(b) Main web page in SA

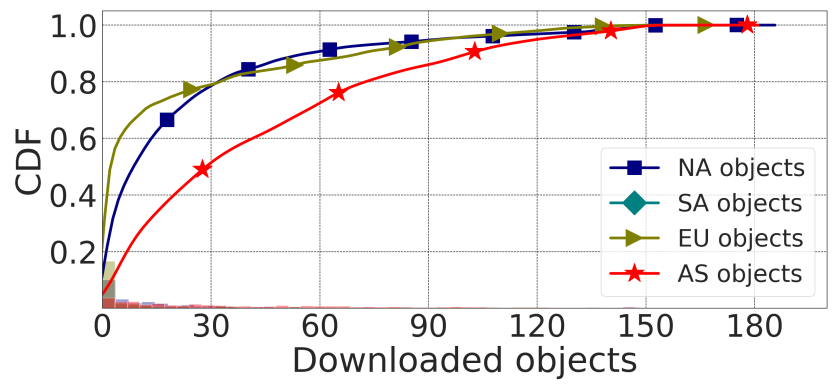

(d) Main web page in AS

Figure 2: Aggregated distribution of resources

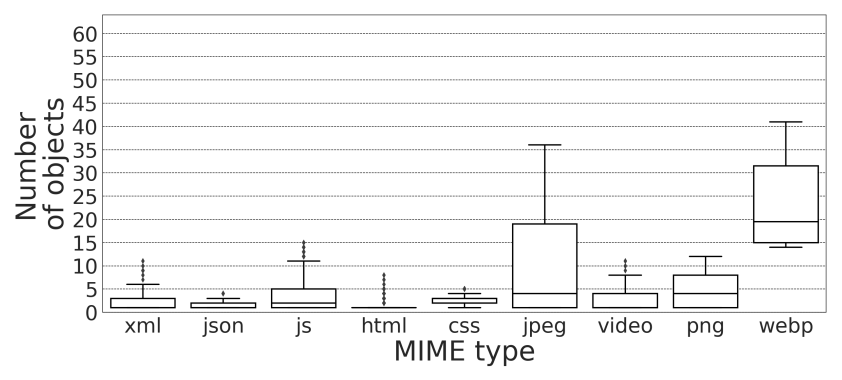

(a) Distribution for Entertainment category

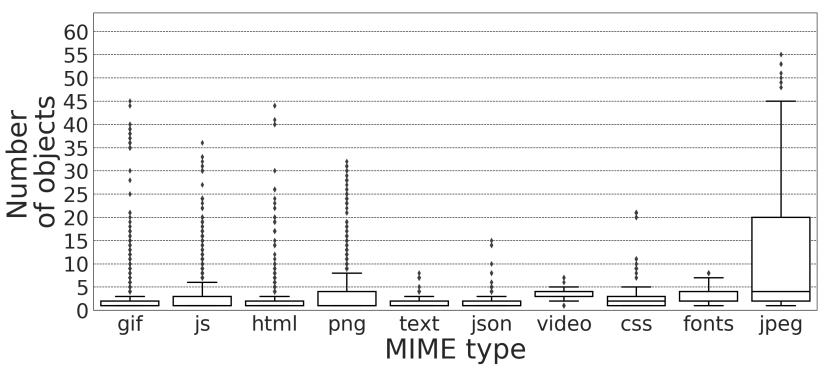

(b) Distribution for Shopping category

Figure 3: MIME types of objects per category

from Asia, and the other half from NA and EU. The mean distributions help in profiling the overall location of the content servers for a European end-user. The Fig. 2 provides the complete distribution of downloaded resources for the 4 continents where the main home page is. The Fig. 2 a shows that when a main web page is located in North America, for $50 \%$ of the measurements, more than 8 objects are downloaded from EU and 50 objects from NA. On the other hand, the Fig. $2 \mathrm{~d}$ shows that when a main web page is in Asia, for $75 \%$ of the different measurements, up to 65 objects are downloaded from AS, 20 objects from EU and 27 objects from NA. We can observe that for a European end-user, many objects are downloaded from Europe, which is due to Content Delivery Networks. For main web pages located in Asia, we can detect that many resources are coming from Asia, which could impact the quality, because of the network delay.

Following the question about what these objects are, Fig. $1 \mathrm{~b}$ represents the breakdown of the downloaded objects by content MIME types of 8 random websites belonging to different categories as referenced by Alexa. While images occupy most of the time the highest distribution type of resources, on average web pages (except Search-Engines category) are composed of 4 css, 5 scripts, 16 images and $2 \mathrm{xml}$. We identified that the average number of scripts and images has increased by $53 \%$ over the last 15 years from past studies [42] and by $7 \%$ from recent studies [39]. Furthermore when paying particular attention to the different types of images, when using a Google-Chrome web browser and visiting a Google website, on average $80 \%$ of images are now in WebP format. Compared to studies conducted in $2011-$ 2014, Flash usage has been reduced by $61 \%$ as Adobe will remove all support for Flash in 2020. When performing our measurements, we also noticed an increasing PWA (Progressive Web Apps) usage of 6\% between July 2018 and January 2019. On a wider scope, based on the Alexa websites Category listings available, 3921 websites were assessed. The News websites download on average $19.81 \%$ more resources, 


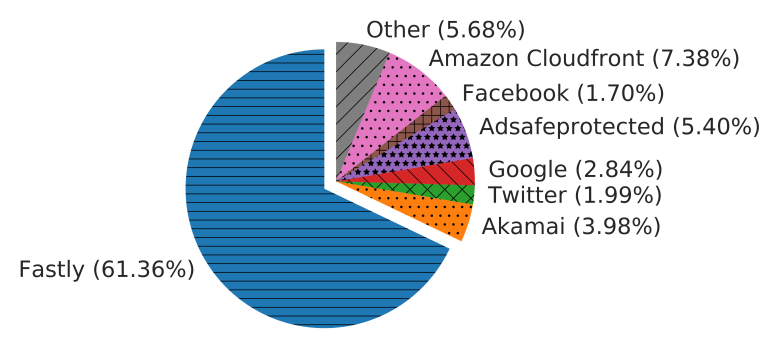

Figure 4: Website lemonde.fr content servers

Kids and Teens websites have a significant greater fraction of Flash objects and Shopping websites make greater usage of JavaScript. When assessing the overall distribution of resources aggregated by MIME type, the Fig. 3 represents two different Alexa-referenced categories where a large variety of objects are incorporated in the corresponding web pages. We selected these 2 categories, since they represent a major part of the popular websites. The Fig. 3a shows that Entertainment websites make greater usage of images (in WebP, $P N G$ or $J P G$ format), followed by JavaScripts and Videos (which can be further decomposed in different formats, e.g WebM, Mp4 or Mp2t). The Shopping websites (Fig. 3b) also make use of a high number of images in different formats which greatly change all along the day thanks to JavaScript.

\subsection{Who is delivering the resources?}

In this analysis, we aimed to identify to which extent, when an end-user navigates to a specific website from a given location, the resources needed are downloaded from many servers located at various places. For this, we analyzed if the contents are downloaded from the same authoritative DNS name server of the main web page. Domains delivering contents and having the same authoritative DNS name server as the main web page are entitled Same-Origin domains and conversely Non-Origin domains. From our global measurements, when a main web page domain is in North America or Europe, irrespective of the preferred Internet protocol, contents are served on average by 2 Same-Origin domains and 7 Non-Origin domains and when the main web page is in Asia or South America, contents are delivered on average by 3 Same-Origin domains and 13 Non-Origin domains. Those Non-Origin domains represent specific services involved in the web page composition (e.g., Google services, such as advertisements or analytics) but also CDN nodes providing contents on behalf of the origin servers.

The average number of domains is not huge, but we can have some websites with many different domains involved in the delivery. As an example of such a complex website, Fig. 4 points out the servers delivering contents when browsing the website lemonde.fr, where the main web page is located in Europe and belongs to the News category. For this website, 352 resources are downloaded from 29 different domains, where 15 domains located in North America deliver 50 resources and 14 domains in Europe deliver 302 resources. The main web page as well as the 13 Same-Origin domains are hosted in Europe by Fastly serving $61.36 \%$ of

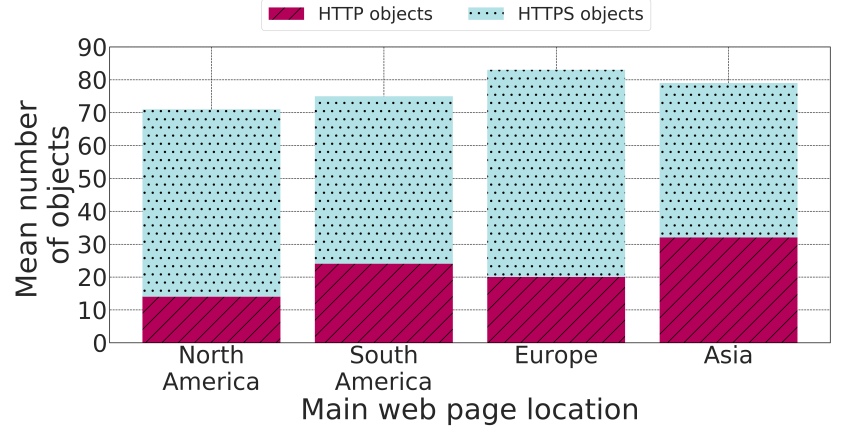

Figure 5: Objects downloaded in HTTP or HTTPS

the overall number of resources, while Akamai and Amazon serve $11.36 \%$ of the resources. The Other content servers regroup 9 different Non-Origin domain web servers. Our website WebView ${ }^{13}$ proposes a graphical representation of this kind of analysis.

\subsection{Do websites secure connections ?}

Compared to the previous studies [43, 44, 45] encryption has been largely adopted to preserve privacy. Indeed, web browsers nowadays favor it by adding by default $h t t p s: / /$ when a user requests a web page. Furthermore, since Google marked non-HTTPS websites as insecure in its Chrome browser in July 2018, HTTPS adoption has increased. However, from our Top 10,000 Alexa websites, only $36.02 \%$ of the websites deliver their contents in full HTTPS (100\% HTTPS) and still $0.28 \%$ of the websites deliver their contents in full HTTP $(100 \%$ HTTP, no resource composing the web page is delivered by an HTTPS server). In between, we have websites composed of resources received with HTTPS and HTTP. As per Fig.5, on average, when the web page is located in North America, 12 resources are downloaded in HTTP and 70 resources in HTTPS and when the main web page is in Asia, 32 resources are downloaded in HTTP and 46 resources in HTTPS. While HTTP/2 and QUIC-enabled web servers mainly deliver contents in HTTPS through TLS $1.2^{14}$, some content servers still deliver resources in a nonsecure mode (HTTP). Those websites are mainly located in Asia.

\subsection{Which Internet protocol do I receive?}

Since the previous published papers analysing Web browsing, the new Internet Protocols, namely HTTP/2 and QUIC, have been promoted. We then wanted to evaluate the adoption of these protocols by web servers. We made measurements explicitly requesting these protocols and analyzed if the remote servers reply using them or if they fall back to another one. Fig. 6 and Fig. 7 show the results of those measurements, in terms of protocol distribution.

First and very logically, we note that when requesting

\footnotetext{
${ }^{13} \mathrm{https}: / /$ webview.orange.com/d/UyIIcrUmz

${ }^{14}$ Now TLS 1.3 is deployed but at the time of the measurements, it was still TLS 1.2
} 


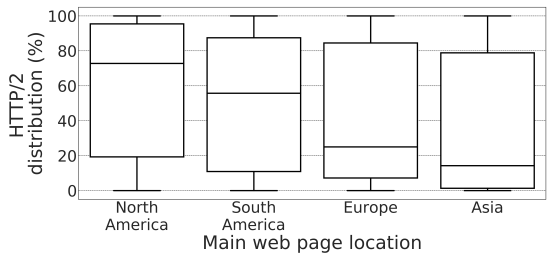

(a) Requesting HTTP/2

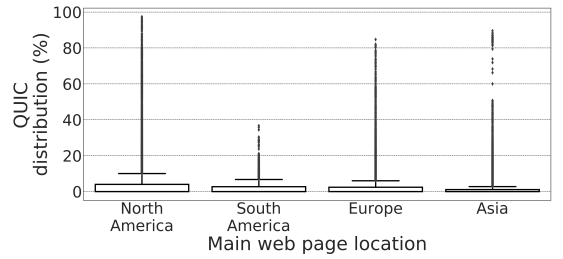

(b) Requesting QUIC

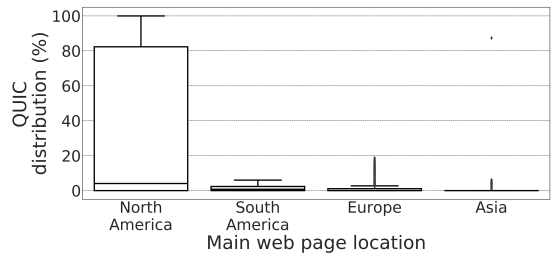

(c) Requesting QUIC Repeat

Figure 6: Received protocol distribution upon request

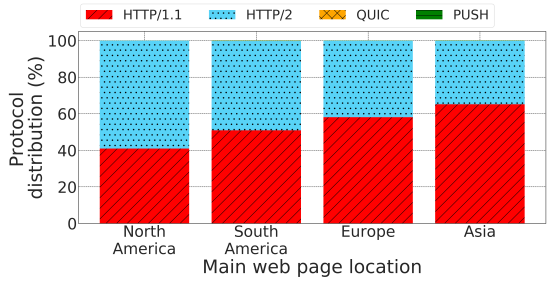

(a) Requesting HTTP/2

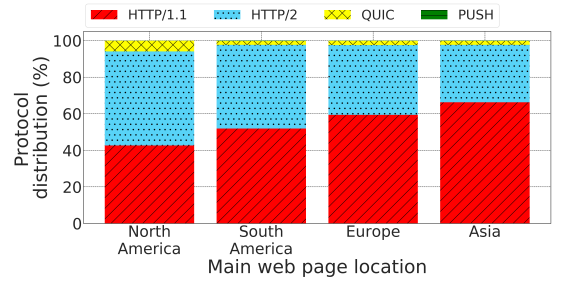

(b) Requesting QUIC

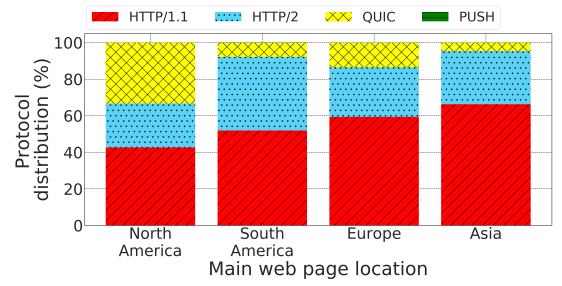

(c) Requesting QUIC Repeat

Figure 7: Average received protocol distribution

web pages with HTTP/1.1, all the servers reply with HTTP/1.1. When performing measurements requesting HTTP/2, we received all the resources in HTTP/2 $(100 \% \mathrm{H} 2)$ only for $11.82 \%$ of the websites. The other $88.18 \%$ of the websites reply in an average protocol distribution of 52.04\% HTTP/1.1, 46.75\% HTTP/2 and $1.21 \%$ Server Push. We can then see that although standardized in 2015, HTTP/2 is not yet widely deployed and that it is not equivalent worldwide, more used for servers in NA or SA, whereas in Europe and Asia, HTTP/1.1 is still prevalent.

When performing tests requesting QUIC, we see that QUIC is not deployed by many servers, but mainly Google ones. On average $97 \%$ of the different QUIC-enabled web servers are Google servers replying in QUIC v.43 when using GoogleChrome v. $68^{15}$ and the other $3 \%$ are non-Google web servers replying in QUIC v.37-38 when using Google-Chrome v. $63^{16}$. From our measurements, we analyze that $0 \%$ of the websites reply in full QUIC for all the resources (100\% HQ) and that the responses are received at an average distribution of 6.12\% QUIC, 50.57\% HTTP/2, 42.1\% HTTP/1.1 and $1.21 \%$ Server Push. QUIC responses are highest when the main web page is in North America (in particular from Google servers).

QUIC is natively used jointly with HTTP/2 for the first request, but offers a QUIC zero-RTT connection when connecting to an already known website. We then evaluate if the distribution is different with the QUIC Repeat mode. In this configuration, $7.21 \%$ of the websites fully reply in QUIC (100\% HQ). The rest of websites, $92.79 \%$, reply in a distribu-

\footnotetext{
15 android.com (90.6\% HQ), google.com (52.6\% HQ) or youtube.com (49.1\% HQ)

${ }^{16}$ facenama.com (81\% HQ), sarkariexam.com (57.4\% HQ) or digitalgrant.ru (55\% HQ)
}

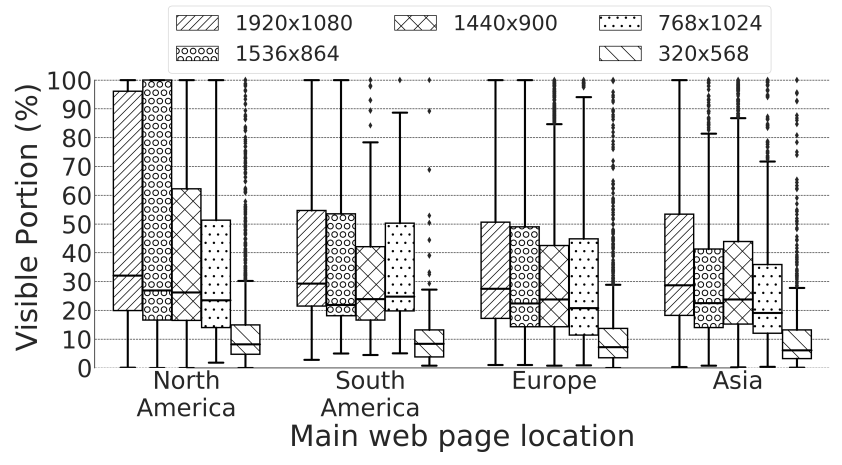

Figure 8: Visible portion of website

tion of 12.71\% QUIC, 45.11\% HTTP/2, 41.09\% HTTP/1.1 and $1.09 \%$ Server Push. We can then see that QUIC Repeat favors QUIC reply distribution, because the browser knows that the website can reply in QUIC.

To sum up this evaluation, HTTP/1.1 is still widely used by web servers, HTTP/2, although standardized in 2015, is deployed at a low pace, and QUIC is mainly used by Google web servers.

\section{Factors impacting Web Browsing Quality}

An indicator of end-users' perceived quality when performing web browsing is the time needed to load a web page (entirely or certain parts of it). This section points out the different factors impacting (decreasing or increasing) these loading times. 


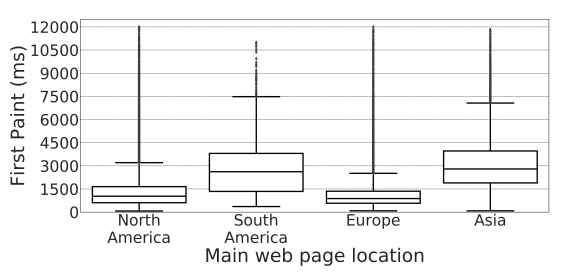

(a) Requesting HTTP/1.1

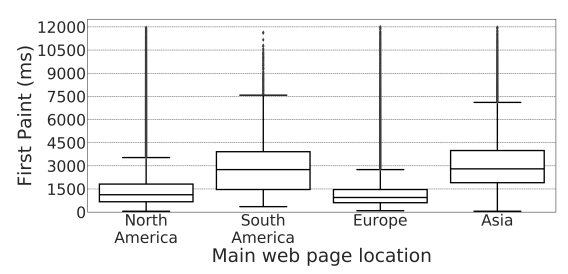

(b) Requesting HTTP/2

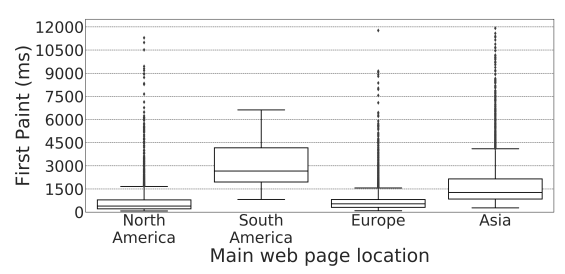

(c) Requesting QUIC Repeat

Figure 9: First Paint loading time

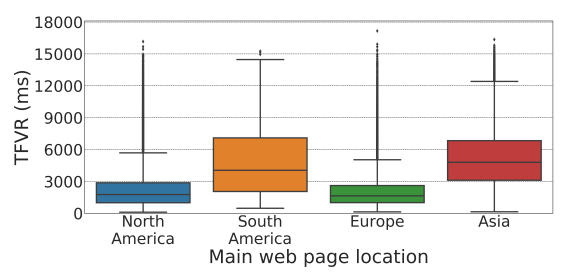

(a) Requesting HTTP/1.1

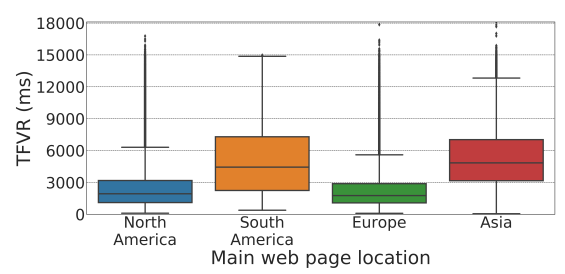

(b) Requesting HTTP/2

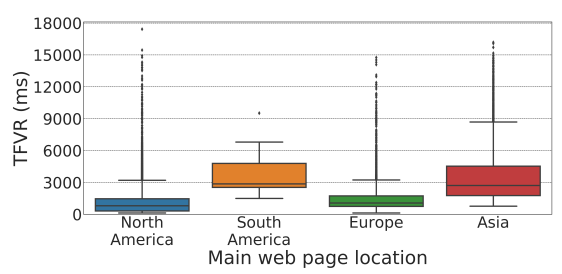

(c) Requesting QUIC Repeat

Figure 10: TFVR loading time

\subsection{Impact of the requested protocol}

We present in this section the impact of the Internet Protocol on the end-users' quality, measured either by the FP, TFVR or PLT. The TFVR provides the loading time of the visible portion of the web page and is tightly linked to the end-user's browser window size. Fig. 8 depicts the different visible portion of web sites (without scrolling the web page) upon different browser window sizes. For example, when the main web page is located in North America, with browser window size of $1920 \times 1080$, less than $50 \%$ of the measurements provide a visible portion less than $32.09 \%$. The Page Load Time provides to an end-user the needed time to load the entire web page (visible and non visible parts).

The FP exposes the moment when a first pixel appears on an end-user's browser screen. The FP involves the download and rendering of the main web page $\mathrm{html}$ source code and on average involves the download of 2 objects from 1 domain for $99.98 \%$ of all measurements. The only difference among the measurements is in general the size of the html page itself, thus increasing the corresponding download time.

As shown in Fig.9a, we can see that the FP value is very close for HTTP/1.1, HTTP/2 and QUIC. This can be explained since before FP, on average only 2 objects are downloaded in this time and all the benefits of the HTTP/2 protocol (multiplexing, header compression, server push func- tion) can not happen. Similarly, for QUIC, since the first request is sent using HTTP/2 and the number of objects very small, 99.76\% of QUIC measurements receive HTTP/2 replies. But one can see that the FP is smaller for the QUIC Repeat mode, since in this case, resources are downloaded in 0-RTT from UDP-enabled web servers and in 1-RTT from TCP-enabled web servers. The First Paint value is a good indicator of the network state when performing web browsing since objects are mostly downloaded from the main web page domain itself thus decreasing the total DNS time.

Looking at Fig.10 and Fig.11, we can see that the behavior for the TFVR and PLT is close to the one detected with the FP measurement, i.e.; there is no noticeable difference between HTTP/1.1, HTTP/2 and QUIC. Indeed, as depicted in Fig.7, when requesting HTTP/2, the HTTP/1.1 Internet protocol is still largely used. Similarly, with our dataset, when requesting QUIC, about $50 \%$ of the measurements have a QUIC distribution less than $0.79 \%$, and those websites fall back to HTTP/2. But for the QUIC Repeat mode, the TFVR is largely smaller, thanks to 0-RTT UDP and 1-RTT TCP. For example, when comparing QUIC Repeat versus HTTP/2, the TFVR loading time is reduced by $54.2 \%$ for a main web page in North America.

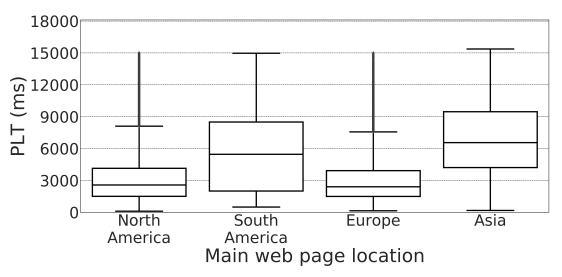

(a) Requesting HTTP/1.1

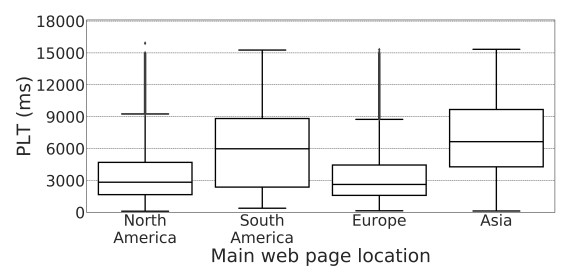

(b) Requesting HTTP/2

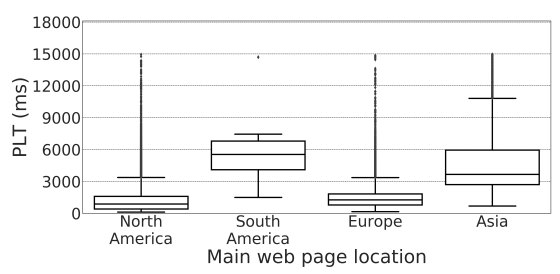

(c) Requesting QUIC Repeat

Figure 11: PLT loading time 
A 6-month Analysis of Factors Impacting Web Browsing Quality for QoE Prediction

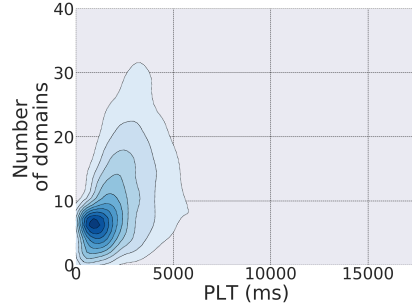

(a) Main web page in NA

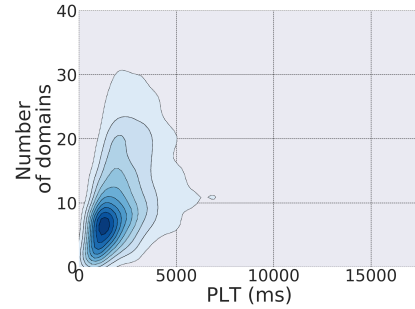

(b) Main web page in EU

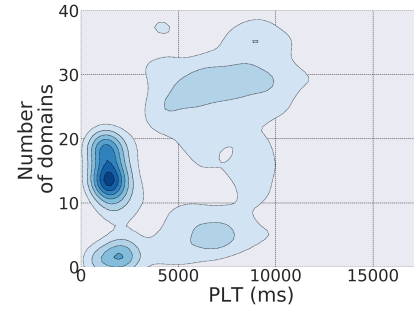

(c) Main web page in SA

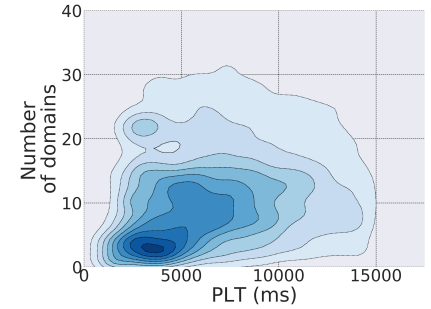

(d) Main web page in AS

Figure 12: Impact of number of domains

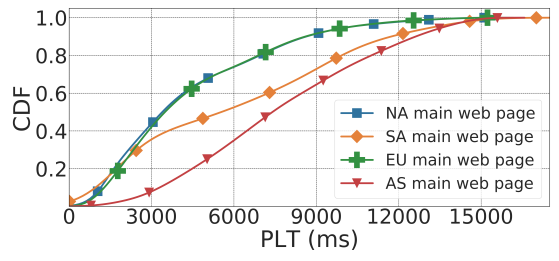

(a) ADSL network access

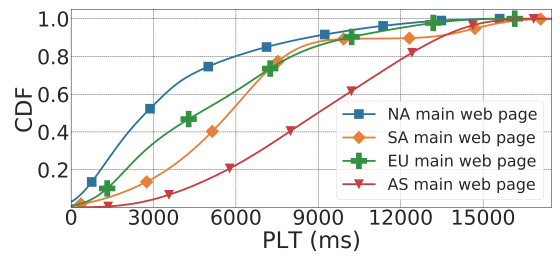

(b) Wi-Fi network access

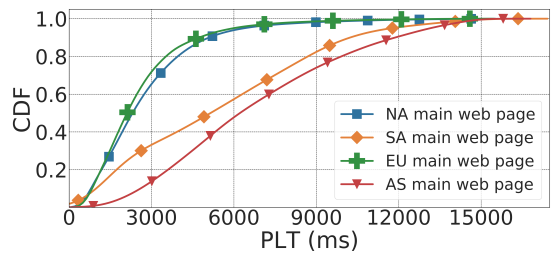

(c) Fiber network access

Figure 13: PLT when requesting HTTP/1.1

\subsection{Impact of the number of domains}

When performing web browsing, the resources are downloaded from various domains (as discussed in section 4.2, from Same-Origin and Non-Origin domains). The number of domains contacted increases proportionally to the DNS lookup times and thus the overall loading times (for the TFVR or PLT). When a web page is located in North America or Europe, on average, resources are downloaded from 9 domains (with an overall DNS time of $316.09 \mathrm{~ms}$ ) and when the main web page is in South America, resources are downloaded on average from 16 domains (with an overall DNS time of $1715.85 \mathrm{~ms}$ ). In general websites being served by large number of domains belong to the News and Shopping category.

Fig.12 illustrates the overall tendency regarding the number of domains through which resources are downloaded when requesting HTTP/2 and the corresponding loading time. This is represented as a heatmap where dark blue represents higher concentration of values and light blue lower concentration of values together with the corresponding Page Load Time. For instance, in Fig.12a, there are plenty of websites having about 7-8 domains and offering a PLT of about $1500 \mathrm{~ms}$, and very few websites having 30 domains for a PLT at about 3000 ms. When a web page is located in North America or Europe, the PLT loading time is good and time increases with the number of domains from which the resources are downloaded. When the main web page is in Asia, the PLT loading time increases proportionally to the number of domains (average of 3915 ms DNS time with the number of domains being 4) and the objects being mainly served in HTTP/1.1 increase blocking and waiting time.

\subsection{Impact of ad blockers}

Advertisement in web pages is nowadays naturally embedded and can decrease an end-user's QoE, regarding the overall loading time of a website. From our measurements, when using an ad blocker (Adblock Plus), on average, the number of downloaded resources is reduced by $11.02 \%$. It is decreased by $9.72 \%$ when the main web page is in North America, by $8.43 \%$ in Europe, by $11.84 \%$ in South America and by $14.10 \%$ when the main web page is in Asia.

Websites belonging to the Shopping, Kids and Teens and Games categories have the most significant decrease in the number of downloaded objects and the PLT for websites belonging to these 3 categories are on average decreased by $39.96 \%$. Regarding other website categories, blocked objects are mainly characterized as social network contents (e.g Facebook, Twitter, LinkedIn, etc.).

\subsection{Impact of the network access}

End-users may be served by different network service providers, along with different bandwidths and data communication technologies (e.g ADSL, Wi-Fi, Fiber). Our measurements reflect observed web browsing quality where

$$
\text { Bandwidth }_{\mathrm{ADSL}}<\text { Bandwidth }_{\mathrm{Wi-Fi}}<\text { Bandwidth }_{\mathrm{Fiber}}
$$

The Fig. 13 depicts the observed PLT loading times when requesting HTTP/1.1 following different network access. For example, when the main web page is in North America, less than $80 \%$ of the measurements have a PLT less than 6901 ms for ADSL network access, PLT less than $6000 \mathrm{~ms}$ for Wi-Fi network access and PLT less than $4000 \mathrm{~ms}$ for Fiber network access, thus representing a reduction of the loading time of $42.03 \%$ when shifting from ADSL to Fiber network access. When requesting the QUIC protocol, as shown in Fig.14, the end-user's QoE increases proportionally to bandwidth increase, independently of the main web page continent location. On average depending on the network access (ADSL vs Fiber), an end-user's PLT is decreased by $30.25 \%$. 
A 6-month Analysis of Factors Impacting Web Browsing Quality for QoE Prediction

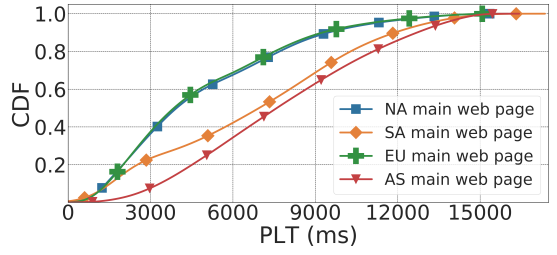

(a) ADSL network access

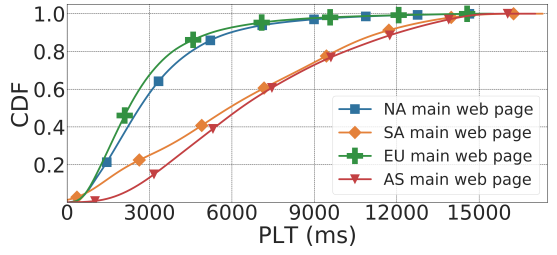

(b) Wi-Fi network access

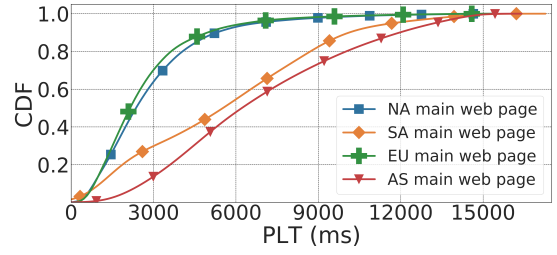

(c) Fiber network access

Figure 14: PLT when requesting QUIC

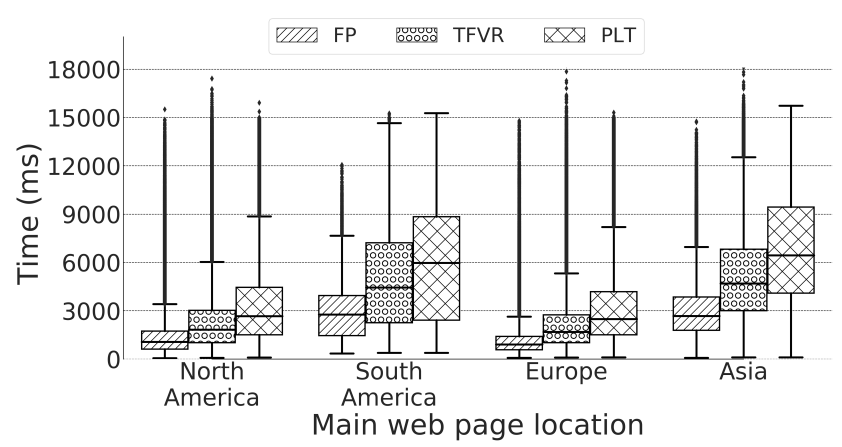

Figure 15: Overall loading times

Increased end-user QoE is tightly linked to the corresponding network access and requested protocol. When requesting HTTP/2 or QUIC, irrespective of the main web page location, web pages' loading times are decreased on average by $19.73 \%$ from ADSL to Wi-Fi, $16.02 \%$ from Wi-Fi to Fiber and $30.25 \%$ from ADSL to Fiber.

\subsection{Impact of main web page location}

Fig. 15 depicts the overall web pages' loading times, grouped by estimated continents location of the home page. The overall centered (median) time follows the rule $F P<T F V R<$ $P L T$. Being in France when performing those measurements, we noticed that the overall loading times for a European end-user are smaller for the websites located in North America and Europe than for those located in South America and Asia. The $25^{\text {th }}$ percentile and $75^{\text {th }}$ percentile are denoted by $Q 1$ and $Q 3$.

When the main web page is located in North America, less than $50 \%$ of the measurements have a PLT less than 2962 ms, $Q 1$ and $Q 3$ are respectively at $1753 \mathrm{~ms}$ and $4868 \mathrm{~ms}$. The parameters inducing the outliers are due to high number of domains (mainly Non-origin), high amount of downloaded objects and objects served over HTTP/1.1. The extreme outliers are for those objects downloaded from South America and Asia. When the main web page is located in South America and Asia, the observed times are higher in general but the number of outliers reduced since more resources are downloaded from the same continent of the main web page domain and the upper limit closer to the defined timeout for every measurement.

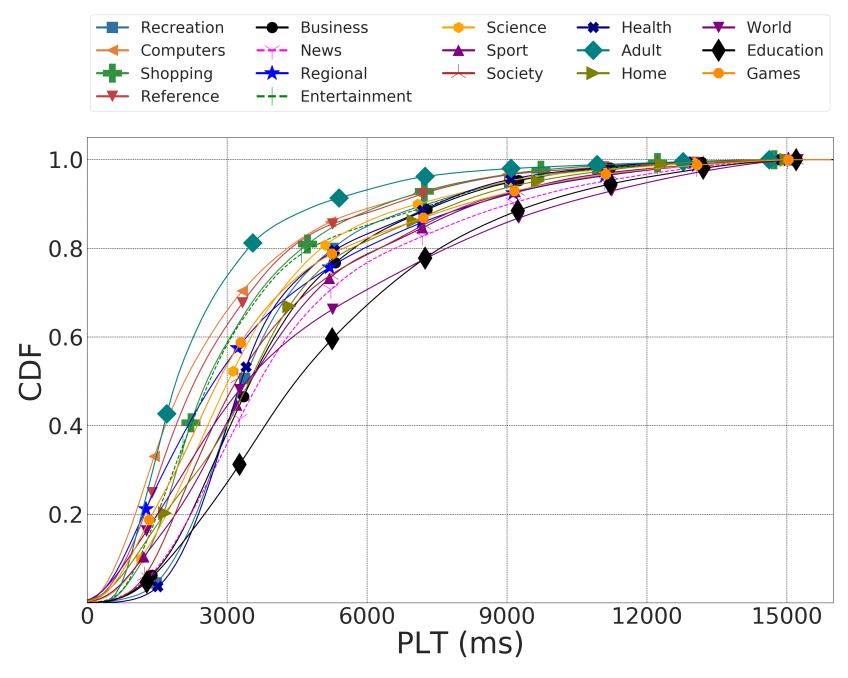

Figure 16: PLT for different websites' categories

\subsection{Impact of websites' referenced categories}

Websites are all different among them composed of a wide range and number of objects. Following their content, they are referenced as belonging to different types of categories (e.g Alexa ${ }^{17}$, QuantCast [46] or Web Filter ${ }^{18}$ ). In our study, we make use of Alexa referenced category listing where websites are classified upon 17 different categories. The Fig. 16 shows the overall observed PLT for different categories. We can observe that the CDF is similar for many categories except for 2 of them (Adult and Education). For instance, for most categories, for $50 \%$ of our measurements, the observed PLT is about 2500-3000 ms. Websites belonging to the Computers, Reference and Shopping category have loading times being close among them (compared to websites belonging to the Recreation, Business and News category) mainly due to objects MIME types composing the web page. There is the biggest gap between the Adult and Education category. While for less than $75 \%$ of our measurements, websites belonging to the Adult category have a PLT less than 3054 ms, websites belonging to the Education category have a PLT less than $6816 \mathrm{~ms}$. While these two categories download on average the same types of objects (mainly images and videos), from our analysis in section 4.4 and 4.1, the main difference which results in a higher observed PLT is that objects for Adult categories are mainly downloaded

\footnotetext{
${ }^{17}$ https://www.alexa.com/topsites/category

${ }^{18} \mathrm{https}$ ///fortiguard.com/webfilter/categories
} 
A 6-month Analysis of Factors Impacting Web Browsing Quality for QoE Prediction

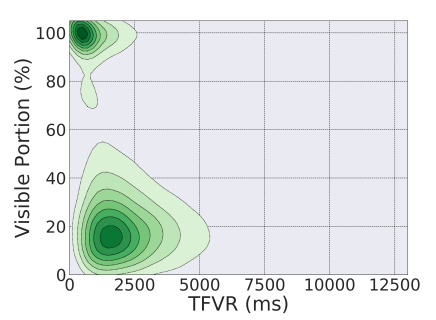

(a) Main web page in NA

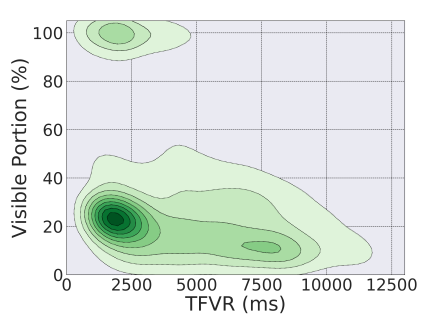

(b) Main web page in SA

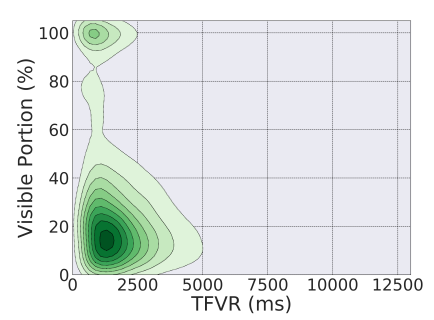

(c) Main web page in EU

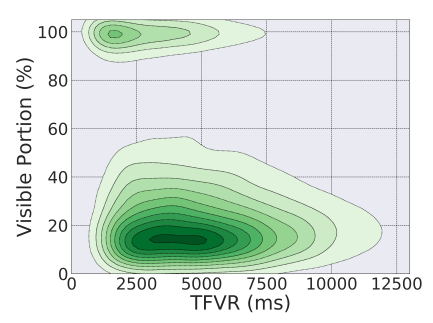

(d) Main web page in AS

Figure 17: Impact of visible portion on TFVR

in HTTP/2 protocol (HTTP/1.1 for Education category) and that images and videos for the Education category are bigger in size.

We can then see that the category can play a role in the web browsing quality, but it is limited and less than the sizes of downloaded objects and the protocol through which they are downloaded which contribute most of the time to a higher PLT value.

\subsection{Impact of visible portion on its corresponding loading time}

Our measurements have been performed following different web browsers' window sizes and the Fig. 8 has shown that depending on the main web page continent location and corresponding web browser window size, an end-user might have a larger overview of the web page without scrolling. We focus in this section on the impact of this visible portion on the time to load it. The default logical assumption would be that the time to load $\lambda \%$ of a web page would be proportional. The Fig. 17 (Heatmap : Dark green represents higher concentration of values and light green lower concentration of values together with the corresponding TFVR) depicts the concentration of websites following their visible portion (without scrolling the web page) and corresponding time to be loaded (TFVR). As discussed in section 4.4, websites having their main web page in North America share the particularity of having higher visible portions (mainly Search-Engines) and the Fig. 17a show that the corresponding TFVR ranges from $400 \mathrm{~ms}$ to $700 \mathrm{~ms}$ when the visible portion is $100 \%$ and ranges from $1200 \mathrm{~ms}$ to $1875 \mathrm{~ms}$ when the visible portion is between $11 \%$ and $20 \%$. The Fig. $17 \mathrm{~d}$ depicts websites whose main web page is in Asia, where we can see that these web pages have a visible portion massively ranging from $13 \%$ to $17 \%$ with loading times between 2700 $\mathrm{ms}$ and $5000 \mathrm{~ms}$ (apart from a limited number of web pages having a visible portion of $100 \%$ and TFVR between 1815 $\mathrm{ms}$ and $2104 \mathrm{~ms}$ ).

The TFVR is not proportional to the visible portion since different actors contribute to this loading time (type and number of objects in the visible portion, the network download time, etc.).

\subsection{Impact of time of day}

Our measurements have been performed round the clock all day long, since following different times of the day a web-

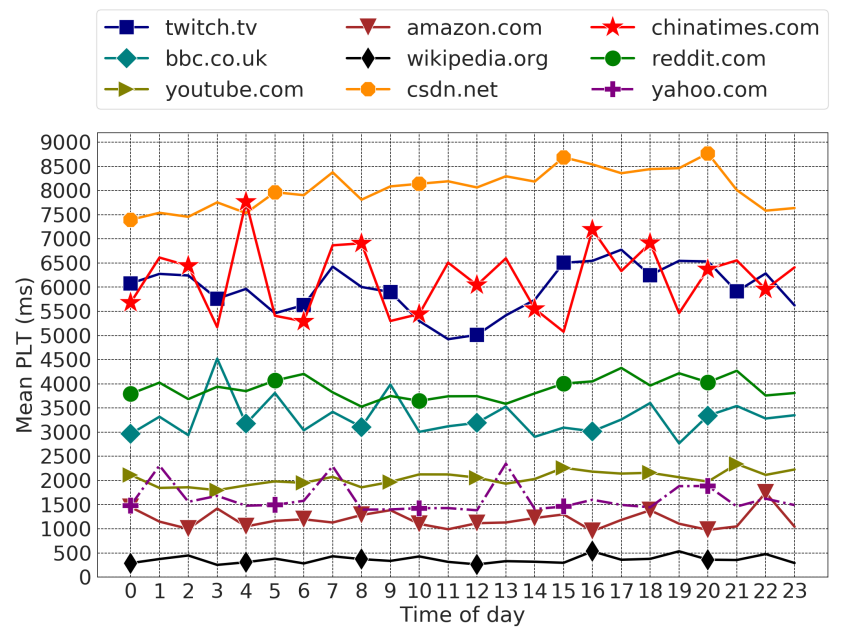

Figure 18: Loading time of different websites at different times of the day

site might be visited by a larger number of end-users, and thus increasing the time needed for the servers to deliver different objects. Furthermore, the overall network state to reach the remote servers might be overloaded (e.g peak hours versus off-peak hours). We thus assess in this section the impact of the time of day on the perceived page load time. The Fig. 18 depicts a set of websites' loading times at different times of the day. While some websites' loading time stay relatively the same all day long (e.g wikipedia.org), other websites depending on their category (thus content) may have their inner structure change several times per day (e.g News: chinatimes.com or Entertainment: twitch.tv). Our measurements have been performed in Europe, and we can notice that the website chinatimes.com average PLT increases drastically at $04 \mathrm{H} \mathrm{CET}(12 \mathrm{H}$ in Asia) and $08 \mathrm{CET}(16 \mathrm{H}$ in Asia). We can thus suppose following these times of the day in Asia that the website has a higher visit rate in Asia, and an European end-user experiences the side effects. In overall, we can notice that between 12H CET - 14 CET or 20H CET $22 \mathrm{H} \mathrm{CET}$, the average loading times perceived by end-users also increase which is most of the time due to the network state.

Web pages loading times are impacted following different times of the day mainly due to their visit rate (web servers impacted) and network state (overloaded during peak hours). 


\begin{tabular}{l|c|c|c} 
Parameters & FP & TFVR & PLT \\
\hline \hline Main web page location & $\checkmark$ & $\checkmark$ & $\checkmark$ \\
Requested protocol & & $\checkmark$ & $\checkmark$ \\
Round Trip Time & $\checkmark$ & $\checkmark$ & $\checkmark$ \\
Website category & $\checkmark$ & $\checkmark$ & $\checkmark$ \\
Number of objects & $\checkmark$ & $\checkmark$ & $\checkmark$ \\
Main HTML page size & $\checkmark$ & $\checkmark$ & $\checkmark$ \\
Size of objects & & $\checkmark$ & $\checkmark$ \\
Types of objects & & $\checkmark$ & $\checkmark$ \\
Visible Portion & & & $\checkmark$ \\
Number of domains & & $\checkmark$ & $\checkmark$ \\
Use of ad blocker & & $\checkmark$ & $\checkmark$ \\
Time of day & $\checkmark$ & $\checkmark$ & $\checkmark$ \\
Network access & $\checkmark$ & $\checkmark$ & $\checkmark$
\end{tabular}

Table 1

Parameters influencing web browsing quality

The corresponding loading times might also fluctuate depending upon the website category since the number of downloaded objects (and MIME type and size) might increase.

\subsection{Which parameters can influence Web Browsing quality?}

Based on our statistical measurements analysis, the Table 1 represents the different parameters to be taken into consideration when investigating the FP (First Paint), TFVR (Time for Full Visual Rendering) and PLT (Page Load Time) loading times. As per our geographic location (Europe), main web pages located in South America and Asia bring higher loading times (compared to main web pages in North America and Europe). The network access and requested protocol are tightly linked to an increased quality. The number, size and type of objects and the main HTML page size have a strong link with the website's Alexa-referenced category listing (e.g Search Engine category websites are composed of small number of objects of small size, News category websites are longer in scrollHeight, thus composed of many objects and served by a large number of domains, etc.). Finally, other factors like the time of day or the visible portion can have an impact on the quality but at a lower degree.

\section{Machine Learning Techniques to predict web browsing quality}

With our statistical analysis, we are able to know the factors impacting the web browsing quality, but we can not manually identify the thresholds or the values leading to a good or a bad quality. For this, we have applied different Machine Learning techniques on our dataset, namely Clustering (and BiClustering), Covariance or Density estimation, Principal Component Analysis (PCA) and Decision Trees. As identified in the previous sections, the factors influencing QoE are linked together and decision tree is revealed to be the most adequate non-parametric supervised learning method. In our study, decision tree rules are detected from $40 \%$ of the entire dataset that we denote by training dataset and the other $60 \%$ is used to validate the rules, denoted by validation dataset. Indeed, defining rules with only the Top 500 or Top 1,000 Alexa is not efficient due to the diversity of the websites structure in the Top 10,000. The decision tree enables us to predict the estimated end-user quality by applying decision rules which are computed from our dataset.

\subsection{Decision Tree based on satisfaction degrees}

Several studies regarding the MOS (Mean Opinion Score) during web browsing sessions [47, 48, 32, 37] provide visible web page loading times. Table 2 presents the different degrees of satisfaction based on end-user sociological perceived feelings [49] coupled to these MOS studies. Instant response indicates a high responsiveness from the visited web page, Seamless response indicates that the end-user is happy with the overall experience, Average response indicates that the end-user feels the delay for the web page to load acceptable, Critical response indicates that the end-user strongly feels the bad side effects of the long web page loading and Bad response indicates that the end-user is not happy at all and can give up the browsing.

Based on the time values mentioned for these satisfaction degrees and the values of the TFVR for our measurements, we hereafter expose the corresponding sets of rules obtained from Decision Trees applied to our training dataset for the 5 classes. The full obtained decision tree is represented by 184 different nodes.

Instant response. Our measurements do not have any TFVR loading time lower than $100 \mathrm{~ms}$ and make this class unnecessary.

Seamless response. $24.32 \%$ of the training dataset has a TFVR in this range and the main impacting factors for this classification are the main web page located in North America or Europe, a low RTT value for the main web page, QUIC Repeat as requested protocol and a Fiber network access.

Average response. $30.67 \%$ of our training values belongs to this class and the main factors are the main web page located in North America or Europe, the requested Internet protocol being HTTP2, QUIC or QUIC Repeat, a Wi-Fi network access, the use of an ad blocker and RTT value between $18.65 \mathrm{~ms}$ and $101.5 \mathrm{~ms}$.

Critical response. $43.78 \%$ of the training measurements have a TFVR in this class, impacted by the main web page located in Europe, South America or Asia, the visible portion between $22.76 \%$ and $41.39 \%$ (these web pages have a larger scroll height), the objects served by various content servers mainly located in Asia and Wi-Fi or ADSL as network access.

Bad response. $1.23 \%$ of the training dataset is classified as Bad response, mainly because of the use of Wi-Fi network access and HTTP/1.1 as requested protocol.

Once this decision tree has been built based on the training dataset, we evaluated it with the validation dataset. The Table 3 depicts the obtained classification confusion matrix, where the diagonal represents the percentage of measurements correctly predicted. We can see that $84.79 \%$ of the predictions are good, but more than $15 \%$ of the validation dataset is wrongly predicted. Looking at the matrix, the val- 
A 6-month Analysis of Factors Impacting Web Browsing Quality for QoE Prediction

Satisfaction degrees $\mid$ Loading Times (ms)

\begin{tabular}{l|c|}
\hline Instant response & $<100$ \\
Seamless response & $100-1000$ \\
Average response & $1000-3000$ \\
Critical response & $3000-10000$ \\
Bad response & $>10000$
\end{tabular}

Table 2

User satisfaction degrees, based on [49]

\begin{tabular}{|c|c|c|c|c|c|}
\cline { 2 - 6 } \multicolumn{1}{c|}{} & \multicolumn{5}{c|}{ Actual class } \\
\hline $\begin{array}{c}\text { Predicted } \\
\text { class }\end{array}$ & Instant & Seamless & Average & Critical & Bad \\
\hline Instant & 0 & $0.15 \%$ & 0 & 0 & 0 \\
\hline Seamless & 0 & $22.21 \%$ & $1.60 \%$ & 0 & 0 \\
\hline Average & 0 & $1.96 \%$ & $29.01 \%$ & $8.07 \%$ & 0 \\
\hline Critical & 0 & 0 & $0.04 \%$ & $32.35 \%$ & $0.01 \%$ \\
\hline Bad & 0 & 0 & $0.02 \%$ & $3.36 \%$ & $1.22 \%$ \\
\hline
\end{tabular}

Table 3

Classification confusion matrix of the validation dataset based on user satisfaction degrees

ues identified in Table 2 and the real TFVR values, we can say that the Instant Response class is unnecessary (no measurement in this class) and that the prediction error rate for the Critical response class is important (about 10\%) because the loading time range is too wide.

\subsection{Decision Tree based on estimated satisfaction degrees from clustering}

Having seen that the proposed classification of the satisfaction degrees from literature is not optimal for current Web Browsing experience, we decided to define a new one, based on our huge dataset of web browsing measurements on the top 10,000 Alexa web sites. We thus look for the best satisfaction degrees using clustering (K-Means) to identify the different classes. The KMeans algorithm divides a set of samples $X$ into $K$ disjoint clusters $C$, where each cluster is described by the mean $\mu_{j}$ of the different samples in the cluster itself. In order to determine the value $K$, we use the Elbow method where we assess the percentage of explained variance (ratio between-group variance to the total variance) as a function of the number of clusters. As seen in Fig.19, the value 5 seems to be the good Elbow Criterion, and using it, we can identify the 5 clusters representing the different satisfaction degrees, illustrated in Table 4. The first and second satisfaction degrees from Table 2 have been merged into one degree and the Critical response satisfaction degree is distributed and split into two degrees. The estimated satisfaction degrees illustrated in Table 4 are more uniformly distributed, compared to Table 2.

We hereafter describe the impacting factors leading to the classification of one measurement into one class, learned from our training dataset by the decision tree model, represented by 176 different nodes.

Good response. $23.32 \%$ of the training dataset has a TFVR in this range and the main factors are the main web

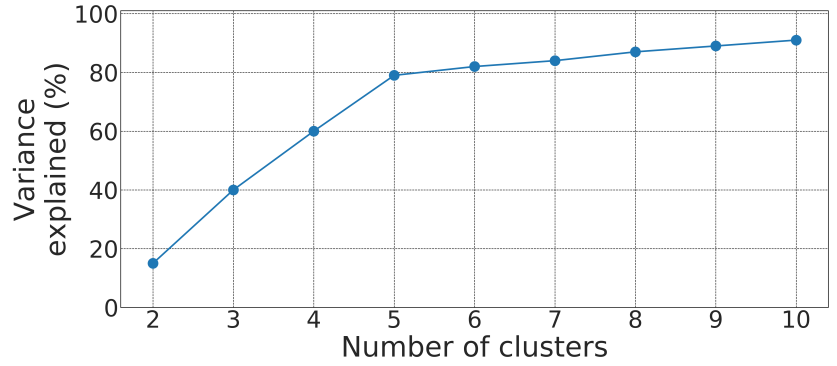

Figure 19: Explained variance

\begin{tabular}{l|c|} 
Estimated satisfaction degrees & Loading Times $\mathbf{( m s )}$ \\
\hline Good response & $<1232$ \\
Fair response & $1232-3486$ \\
Moderate response & $3486-6715$ \\
Worse response & $6715-9281$ \\
Poor response & $>9281$
\end{tabular}

Table 4

Estimated satisfaction degrees derived from our measurements

page located in North America or Europe, a low RTT and number of domains serving contents from North America or Europe, with replies delivered over the QUIC protocol.

Fair response. $27.31 \%$ of the training measurements are classified in this class and the main impacting factors are namely the main web page located in North America, a RTT value less than $105.5 \mathrm{~ms}$, a Wi-Fi or Fiber network access, resources downloaded from North America, Europe or Asia in HTTPS, together with a low number of domains serving contents.

Moderate response. $31.26 \%$ of our training dataset leads to Moderate response, mainly based on the main web page located in Europe or Asia, the requested Internet protocol being HTTP/1.1, HTTP/2 or QUIC, the visible portion of the websites less than $13.25 \%$ and objects delivered from Asia or South America.

Worse response. $12.09 \%$ of the training dataset has a TFVR in this range with the main factors being the main web page located in South America or Asia, the number of downloaded resources between 45 and 85 (mainly downloaded from Asia), RTT value greater than $185.5 \mathrm{~ms}$ and the number of domains from Asia greater than 4.

Poor response. $6.02 \%$ of the training measurements lead to a poor quality, mainly because of the main web page located in Asia, HTTP/1.1 used as requested protocol, a high number of downloaded objects and the use of an ADSL network access.

We evaluate the accuracy of our decision tree with the rest of our dataset (i.e., $60 \%$ of our measurements) and the Table 5 depicts the obtained classification confusion matrix when applying the obtained rules on this validation dataset. We can see, looking at the diagonal representing the percentage of measurements correctly predicted, that our rules can efficiently predict $94.17 \%$ of the dataset. Only $5.83 \%$ of the validation dataset is wrongly predicted, which is 2.61 times 
A 6-month Analysis of Factors Impacting Web Browsing Quality for QoE Prediction

\begin{tabular}{|c|c|c|c|c|c|}
\cline { 2 - 6 } \multicolumn{1}{c|}{} & \multicolumn{5}{c|}{ Actual class } \\
\hline $\begin{array}{c}\text { Predicted } \\
\text { class }\end{array}$ & Good & Fair & Moderate & Worse & Poor \\
\hline Good & $22.19 \%$ & $0.04 \%$ & 0 & 0 & 0 \\
\hline Fair & $1.13 \%$ & $25.17 \%$ & $0.82 \%$ & 0 & 0 \\
\hline Moderate & 0 & $2.10 \%$ & $29.22 \%$ & $0.43 \%$ & 0 \\
\hline Worse & 0 & 0 & $1.20 \%$ & $11.63 \%$ & $0.06 \%$ \\
\hline Poor & 0 & 0 & $0.02 \%$ & $0.03 \%$ & $5.96 \%$ \\
\hline
\end{tabular}

Table 5

Classification confusion matrix of the validation dataset based on estimated satisfaction degrees

\begin{tabular}{|c|c|c|c|c|c|}
\cline { 2 - 6 } \multicolumn{1}{c|}{} & \multicolumn{5}{c|}{ Actual class } \\
\hline $\begin{array}{c}\text { Predicted } \\
\text { class }\end{array}$ & Good & Fair & Moderate & Worse & Poor \\
\hline Good & $6.51 \%$ & $0.16 \%$ & 0 & 0 & 0 \\
\hline Fair & $0.76 \%$ & $21.09 \%$ & $0.72 \%$ & 0 & 0.02 \\
\hline Moderate & $0.01 \%$ & $1.96 \%$ & $35.02 \%$ & $0.12 \%$ & $0.01 \%$ \\
\hline Worse & 0 & 0 & $3.60 \%$ & $17.74 \%$ & $1.03 \%$ \\
\hline Poor & 0 & 0 & 0 & $1.21 \%$ & $10.04 \%$ \\
\hline
\end{tabular}

Table 6

Classification confusion matrix to validate the accuracy of our rules-based model, based on estimated satisfaction degrees

less when compared to the rules-based model obtained in section 6.1. We can then think that this model is better.

\subsection{Accuracy of our rules-based model}

In order to verify the correctness of the obtained decision tree from section 6.2, we have performed in February 2019 measurements on the Alexa websites ranging from rank 10,000 to 15,000 . The dataset sums up to the measurement of 4861 never-assessed before distinct websites, which represent 2.7 Million different measurements. The Table 6 illustrates the obtained classification confusion matrix for these measurements when applying our rules-based model.

Among our measurements, $6.51 \%$ of the measurements provide a Good response (low RTT and small number of objects), $21.09 \%$ for Fair response (low RTT and main web page in Europe), 35.02\% for Moderate response (large number of domains serving contents from Europe and Asia), $17.04 \%$ for Worse response and finally $10.04 \%$ of the measurements yield a Poor response (high HTTP/1.1 reply distribution and objects mainly delivered from South America and Asia). In short, $90.4 \%$ of the overall new dataset was correctly predicted. This is a bit less than with the validation dataset, but it is related to websites which have never been accessed before, in contrast to the validation dataset, composed of websites being also in the training dataset. We can then conclude that our rules-based model can efficiently predict web browsing quality for any website for an end-user located in France.

We have presented in this paper the results of the analysis based on our measurements, performed by the probes in France (Europe). Our model has been proved to be correct for this configuration but it has to be adapted to other situations, e.g., end-user in another location. Indeed, as seen in Fig. 1a and Fig. 2, when visiting different websites having their main web page located in specific continent-geographic locations, resources are downloaded from different continents (e.g for a European end-user visiting a web page in Asia, resources are downloaded from Europe, North America and Asia), which impacts the quality. As depicted in Table. 1, the impact of parameters to be re-assessed will be the Round-Trip-Time (network path taken through different Asynchronous Systems), number and types of objects (mainly due to advertising), the number of domains (specifically the location of web servers delivering content), time of the day and corresponding network access (the throughput offered by network operators from different regions are different).

\section{Conclusion and Future work}

In this paper we have shown the importance of taking into account diverse parameters to better quantify and qualify web browsing. We have used all the web metrics identified by the $\mathrm{W} 3 \mathrm{C}$ or the research community and real onmarket web browsers together with user-representative residential network access. Our study has brought light on the wide range of factors which can improve or decrease an enduser's web browsing quality. The identified impacting factors are then used as inputs to a rule-based model obtained from Decision Tree, in order to efficiently predict the web browsing quality.

Our measurements have been performed in France and an ongoing and future work is focused on performing measurements from different locations and using different network providers all around the world, to confirm the impacting factors to be taken into consideration, to refine the estimated satisfaction degrees for end-users' perceived web browsing quality and to validate our model. The obtained loading times contributing to the different estimated satisfaction degrees will also be assessed through a MOS study with real end-users.

\section{Acknowledgement}

This work is partially funded by the French ANR BottleNet project, No ANR-15-CE25-0013-001.

\section{References}

[1] Z. Abbassi, N. Hegde, L. Massoulié, Distributed content curation on the web, Sigmetrics Performance Evaluation Review (2014).

[2] P. Snyder, L. Ansari, C. Taylor, C. Kanich, Browser feature usage on the modern web, in: Proceedings of the 2016 ACM on Internet Measurement Conference, IMC 2016, Santa Monica, CA, USA, November 14-16, 2016, 2016.

[3] Sandinve, The 2018 Global Internet Phenomena Report, 2018. URL: https://www. sandvine. com/hubfs/downloads/phenomena/ 2018-phenomena-report.pdf.

[4] M. Belshe, R. Peon, M. Thomson, Hypertext Transfer Protocol Version 2 (HTTP/2), RFC 7540, RFC Editor, 2015. URL: http://www.rfc-editor. org/rfc/rfc7540.txt, http://www. rfc-editor. org/rfc/rfc7540.txt.

[5] J. Iyengar, M. Thomson, QUIC: A UDP-Based Multiplexed and Secure Transport, Internet-Draft draft-ietf-quic-transport-15, Internet 
Engineering Task Force, 2018. URL: https://datatracker.ietf.org/ doc/html/draft-ietf-quic-transport-15, work in Progress.

[6] R. Shade, M. Warres, HTTP/2 Semantics Using The QUIC Transport Protocol, Internet-Draft, 2016. URL: https://datatracker. ietf.org/ doc/draft-shade-quic-http2-mapping/.

[7] I. Grigorik, Above the fold time: Measuring web page performance visually, 2012. URL: http://conferences.oreilly.com/ velocity/velocity-mar2011/public/schedule/detail/18692.

[8] A. Saverimoutou, B. Mathieu, S. Vaton, Web browsing measurements: An above-the-fold browser-based technique, in: 38th IEEE International Conference on Distributed Computing Systems, ICDCS 2018, Vienna, Austria, July 2-6, 2018, 2018.

[9] C. Avram, K. Salem, B. Wong, Latency amplification: Characterizing the impact of web page content on load times, in: 33rd IEEE International Symposium on Reliable Distributed Systems Workshops, SRDS Workshops 2014, Nara, Japan, October 6-9, 2014, 2014, pp. 20-25.

[10] M. Malloy, M. McNamara, A. Cahn, P. Barford, Ad blockers: Global prevalence and impact, in: Proceedings of the 2016 ACM on Internet Measurement Conference, IMC 2016, Santa Monica, CA, USA, November 14-16, 2016, 2016.

[11] B. C. Vattikonda, V. Dave, S. Guha, A. C. Snoeren, Empirical analysis of search advertising strategies, in: Proceedings of the 2015 ACM Internet Measurement Conference, IMC 2015, Tokyo, Japan, October 28-30, 2015, 2015.

[12] E. Pujol, O. Hohlfeld, A. Feldmann, Annoyed users: Ads and adblock usage in the wild, in: Proceedings of the 2015 ACM Internet Measurement Conference, IMC 2015, Tokyo, Japan, October 28-30, 2015, 2015.

[13] G. Acar, M. Juárez, N. Nikiforakis, C. Díaz, S. F. Gürses, F. Piessens, B. Preneel, Fpdetective: dusting the web for fingerprinters, in: 2013 ACM SIGSAC Conference on Computer and Communications Security, CCS'13, Berlin, Germany, November 4-8, 2013, 2013, pp. 11291140. doi:10.1145/2508859.2516674.

[14] S. Englehardt, A. Narayanan, Online tracking: A 1-million-site measurement and analysis, in: Proceedings of the 2016 ACM SIGSAC Conference on Computer and Communications Security, Vienna, Austria, October 24-28, 2016, 2016, pp. 1388-1401. doi:10.1145/ 2976749.2978313.

[15] J. R. Mayer, J. C. Mitchell, Third-party web tracking: Policy and technology, in: IEEE Symposium on Security and Privacy, SP 2012, 21-23 May 2012, San Francisco, California, USA, 2012, pp. 413-427.

[16] T. Libert, Exposing the hidden web: An analysis of third-party HTTP requests on 1 million websites, volume abs/1511.00619, 2015.

[17] M. Lécuyer, G. Ducoffe, F. Lan, A. Papancea, T. Petsios, R. Spahn, A. Chaintreau, R. Geambasu, Xray: Enhancing the web's transparency with differential correlation, in: Proceedings of the 23rd USENIX Security Symposium, San Diego, CA, USA, August 20-22, 2014., 2014, pp. 49-64.

[18] A. Datta, M. C. Tschantz, A. Datta, Automated experiments on ad privacy settings, volume 2015, 2015, pp. 92-112.

[19] P. Wang, M. Varvello, A. Kuzmanovic, Kaleidoscope: A crowdsourcing testing tool for web quality of experience, The 39th IEEE International Conference on Distributed Computing Systems (ICDCS 2019) (2019).

[20] M. Seufert, N. Wehner, P. Casas, Studying the impact of HAS qoe factors on the standardized qoe model P.1203, in: 38th IEEE International Conference on Distributed Computing Systems, ICDCS 2018, Vienna, Austria, July 2-6, 2018, 2018.

[21] A. Sackl, P. Casas, R. Schatz, L. Janowski, R. Irmer, Quantifying the impact of network bandwidth fluctuations and outages on web qoe, in: Seventh International Workshop on Quality of Multimedia Experience, QoMEX 2015, Pilos, Messinia, Greece, May 26-29, 2015, 2015, pp. 1-6.

[22] S. Cook, B. Mathieu, P. Truong, I. Hamchaoui, QUIC: Better For What And For Whom?, in: Proceedings of IEEE International Conference on Communications (ICC), 2017.

[23] R. Lychev, S. Jero, A. Boldyreva, C. Nita-Rotaru, How Secure and
Quick is QUIC? Provable Security and Performance Analyses, in: Proceedings of the IEEE Symposium on Security and Privacy (SP), 2015.

[24] J. Manzoor, I. Drago, R. Sadre, How HTTP/2 is changing web traffic and how to detect it, in: Network Traffic Measurement and Analysis Conference, TMA 2017, Dublin, Ireland, June 21-23, 2017, 2017.

[25] J. Ruth, I. Poese, C. Dietzel, O. Hohlfeld, A first look at QUIC in the wild, in: Passive and Active Measurement - 19th International Conference, PAM 2018, Berlin, Germany, March 26-27, 2018, Proceedings, 2018.

[26] A. M. Kakhki, S. Jero, D. R. Choffnes, C. Nita-Rotaru, A. Mislove, Taking a long look at QUIC: an approach for rigorous evaluation of rapidly evolving transport protocols, in: Proceedings of the $2017 \mathrm{In}-$ ternet Measurement Conference, IMC 2017, London, United Kingdom, November 1-3, 2017, 2017.

[27] P. Biswal, O. Gnawali, Does quic make the web faster?, in: 2016 IEEE Global Communications Conference (GLOBECOM), 2016, pp. 1-6. doi:10.1109/GLOCOM. 2016.7841749.

[28] E. Pujol, P. Richter, B. Chandrasekaran, G. Smaragdakis, A. Feldmann, B. M. Maggs, K. Ng, Back-office web traffic on the internet, in: Proceedings of the 2014 Internet Measurement Conference, IMC 2014, Vancouver, BC, Canada, November 5-7, 2014, 2014.

[29] Y. Yang, L. Zhang, R. Maheshwari, Z. A. Kahn, D. Agarwal, S. Dubey, Scout: A point of presence recommendation system using real user monitoring data, in: Passive and Active Measurement 17th International Conference, PAM 2016, Heraklion, Greece, March 31 - April 1, 2016, 2016.

[30] R. Anwar, H. Niaz, D. R. Choffnes, Í. S. Cunha, P. Gill, E. KatzBassett, Investigating interdomain routing policies in the wild, in: Proceedings of the 2015 ACM Internet Measurement Conference, IMC 2015, Tokyo, Japan, October 28-30, 2015, 2015.

[31] U. Naseer, T. Benson, Inspectorgadget: Inferring network protocol configuration for web services, in: 38th IEEE International Conference on Distributed Computing Systems, ICDCS 2018, Vienna, Austria, July 2-6, 2018, 2018.

[32] E. Bocchi, L. D. Cicco, D. Rossi, Measuring the quality of experience of web users, Computer Communication Review (2016).

[33] C. Kelton, J. Ryoo, A. Balasubramanian, S. R. Das, Improving user perceived page load times using gaze, in: 14th USENIX Symposium on Networked Systems Design and Implementation, NSDI 2017, Boston, MA, USA, March 27-29, 2017, 2017.

[34] M. Varvello, J. Blackburn, D. Naylor, K. Papagiannaki, EYEORG: A platform for crowdsourcing web quality of experience measurements, in: Proceedings of the 12th International on Conference on emerging Networking EXperiments and Technologies, CoNEXT 2016, Irvine, California, USA, December 12-15, 2016, 2016, pp. 399-412.

[35] Q. Gao, P. Dey, P. Ahammad, Perceived performance of top retail webpages in the wild: Insights from large-scale crowdsourcing of above-the-fold qoe, in: Proceedings of the 2017 Workshop on QoEbased Analysis and Management of Data Communication Networks, Internet-QoE@SIGCOMM 2017, Los Angeles, CA, USA, August 21, 2017, 2017.

[36] T. Enghardt, T. Zinner, A. Feldmann, Web performance pitfalls, in: D. Choffnes, M. Barcellos (Eds.), Passive and Active Measurement, Springer International Publishing, Cham, 2019, pp. 286-303.

[37] D. N. D. Hora, A. S. Asrese, V. Christophides, R. Teixeira, D. Rossi, Narrowing the gap between qos metrics and web qoe using above-thefold metrics, in: Passive and Active Measurement - 19th International Conference, 2018.

[38] A. Saverimoutou, B. Mathieu, S. Vaton, Web view: Measuring and monitoring representative information on websites, in: IEEE International Workshop on Quality of Experience Management, QOEMANAGEMENT Paris, France, February 18, 2019, 2019.

[39] M. Butkiewicz, H. V. Madhyastha, V. Sekar, Characterizing web page complexity and its impact, IEEE/ACM Trans. Netw. 22 (2014) 943956.

[40] M. Butkiewicz, H. V. Madhyastha, V. Sekar, Understanding website complexity: measurements, metrics, and implications, in: Proceed- 
ings of the 11th ACM SIGCOMM Internet Measurement Conference, IMC'11, Berlin, Germany, November 2-, 2011, 2011, pp. 313-328.

[41] S. Ihm, V. S. Pai, Towards understanding modern web traffic, in: Proceedings of the 11th ACM SIGCOMM Internet Measurement Conference, IMC '11, Berlin, Germany, November 2-, 2011, 2011, pp. 295-312.

[42] F. Hernández-Campos, K. Jeffay, F. D. Smith, Tracking the evolution of web traffic: 1995-2003, in: 11th International Workshop on Modeling, Analysis, and Simulation of Computer and Telecommunication Systems (MASCOTS 2003), 12-15 October 2003, Orlando, FL, USA, 2003, pp. 16-25.

[43] A. P. Felt, R. Barnes, A. King, C. Palmer, C. Bentzel, P. Tabriz, Measuring HTTPS adoption on the web, in: 26th USENIX Security Symposium, USENIX Security 2017, Vancouver, BC, Canada, August 1618, 2017., 2017, pp. 1323-1338.

[44] K. Krombholz, W. Mayer, M. Schmiedecker, E. R. Weippl, "i have no idea what i'm doing" - on the usability of deploying HTTPS, in: 26th USENIX Security Symposium, USENIX Security 2017, Vancouver, BC, Canada, August 16-18, 2017., 2017, pp. 1339-1356.

[45] G. Michaelson, M. Roughan, J. Tuke, M. P. Wand, R. Bush, Rigorous statistical analysis of HTTPS reachability, CoRR abs/1706.02813 (2017).

[46] S. Simpson, Interest Category Definitions, 2018. URL: https://help.quantcast.com/hc/en-us/articles/ 115014006128-Interest-Category-Definitions.

[47] T. Hobfeld, F. Metzger, D. Rossi, Speed index: Relating the industrial standard for user perceived web performance to web qoe, in: Tenth International Conference on Quality of Multimedia Experience, 2018.

[48] E. Bocchi, L. D. Cicco, M. Mellia, D. Rossi, The web, the users, and the MOS: influence of HTTP/2 on user experience, in: Passive and Active Measurement - 18th International Conference, PAM 2017, Sydney, NSW, Australia, March 30-31, 2017, Proceedings, 2017.

[49] J. Nielsen, Web response times, 2018. URL: https://www. nngroup. com/articles/website-response-times/.

\section{APPENDIX}

\begin{tabular}{|c|c|}
\hline Indicators & \\
\hline wwwName & Assessed website name \\
\hline ranking & Alexa referenced ranking \\
\hline preferredProtocol & The requested protocol \\
\hline addBlock & The use (or not) of an adblocker \\
\hline netwlface & $\begin{array}{l}\text { The used network interface } \\
\text { (ADSL, Wi-Fi, Fiber) }\end{array}$ \\
\hline timeDay & Time measesurement performed \\
\hline browserWidth & Width of the used browser \\
\hline browserHeight & Height of the used browser \\
\hline pingValue & Round Trip Time upon main web page \\
\hline visiblePortion & $\begin{array}{l}\text { Visible portion of the web page at first } \\
\text { glance(no scrolling) }\end{array}$ \\
\hline mainWWWLocation & Main web page continent location \\
\hline h1Share & HTTP/1.1 overall replies distribution \\
\hline h2Share & HTTP/2 overall replies distribution \\
\hline hqShare & QUIC overall replies distribution \\
\hline pushShare & SERVER PUSH replies distribution \\
\hline nbRess & Number of downloaded objects \\
\hline nbObjNA & $\begin{array}{l}\text { Number of objects downloaded from } \\
\text { North America }\end{array}$ \\
\hline nbObjSA & $\begin{array}{l}\text { Number of objects downloaded from } \\
\text { South America }\end{array}$ \\
\hline nbObjEU & $\begin{array}{l}\text { Number of Objects downloaded from } \\
\text { Europe }\end{array}$ \\
\hline nbObjOrigin & $\begin{array}{l}\text { Number of objects downloaded from } \\
\text { Same-Origin server }\end{array}$ \\
\hline nbObjNonOrigin & $\begin{array}{l}\text { Number of objects downloaded from } \\
\text { Non-Origin server }\end{array}$ \\
\hline nbDomains & Number of domains serving contents \\
\hline nbDomNA & Number of domains in North America \\
\hline nbDomEU & Number of domains in Europe \\
\hline nbDomAS & Number of domains in Asia \\
\hline h1ShareBFP & $\begin{array}{l}\text { HTTP } / 1.1 \text { replies distribution } \\
\text { before the First Paint }\end{array}$ \\
\hline h2ShareBFP & $\begin{array}{l}\text { HTTP } / 2 \text { replies distribution } \\
\text { before the First Paint }\end{array}$ \\
\hline hqShareBFP & $\begin{array}{l}\text { QUIC replies distribution } \\
\text { before the First Paint }\end{array}$ \\
\hline pushShareBFP & $\begin{array}{l}\text { SERVER PUSH replies } \\
\text { distribution before the First Paint }\end{array}$ \\
\hline h1ShareBTFVR & $\begin{array}{l}\text { HTTP/1.1 replies distribution } \\
\text { before the TFVR }\end{array}$ \\
\hline h2ShareBTFVR & $\begin{array}{l}\text { HTTP } / 2 \text { replies distribution } \\
\text { before the TFVR }\end{array}$ \\
\hline hqShareBTFVR & $\begin{array}{l}\text { QUIC replies distribution } \\
\text { before the TFVR }\end{array}$ \\
\hline$\%$ Obj before FP & $\begin{array}{l}\text { Percentage of objects downloaded } \\
\text { before FP }\end{array}$ \\
\hline$\%$ Obj before TFVR & $\begin{array}{l}\text { Percentage of objects downloaded } \\
\text { before TFVR }\end{array}$ \\
\hline categoryType & Website category referenced by Alexa \\
\hline
\end{tabular}

Table 7

Decision Tree main factors' acronyms 